\title{
Vertrauen im Islam - Misstrauen gegen den Islam
}

Rifa'at Lenzin

Vertrauen im Islam bedeutet im islamischen Kontext in erster Linie und vor allem Vertrauen in Gott, arabisch tawakkul. Das ist insofern naheliegend, als der Begriff "Islam" Hingabe [gemeint an Gott] bedeutet. Einer solchen Aufforderung der absoluten und freiwilligen Hingabe an Gott wird nur Folge leisten können, wer auch absolutes Vertrauen in diesen Gott hat. Einige Attribute Gottes beziehen sich denn auch auf diesen Aspekt, z.B. al-wakīl, der Vertrauenswürdige, aber auch al-waliyy, der Schutzherr eines jeden, der Seinen Schutz und Seine Leitung braucht. Das Vertrauen in Gott tawakkul 'alā -llāh ist ein fundamentaler Bestandteil des Glaubens als solchen (im Qur'an als imān bezeichnet).

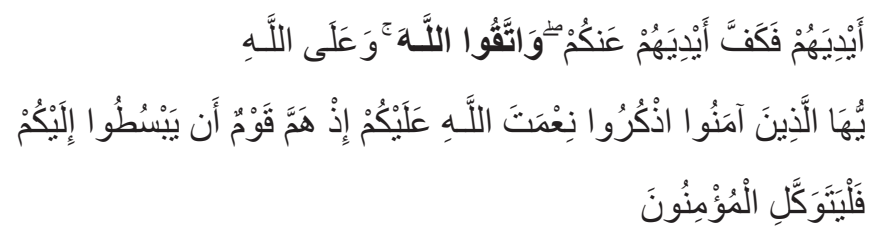

O ihr, die ihr glaubt! Gedenkt der Gnade Allahs über euch, als eine Gruppe die Hände nach euch auszustrecken trachtete. Er aber hielt ihre Hände von euch zurück. Und fürchtet Allah; auf Allah sollen die Gläubigen vertrauen. (Q. 5,11)

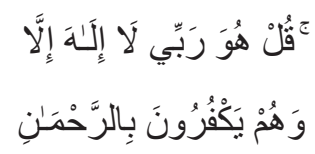

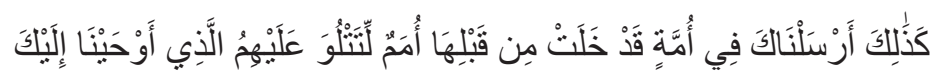

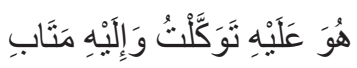

Und so haben Wir dich zu einem Volk gesandt, vor dem bereits andere Völker dahingegangen sind, auf dass du ihnen verkünden mögest, was Wir dir offenbarten; und doch glauben sie nicht an den Allerbarmer. Sprich:"Er 
ist mein Herr; kein Gott ist da außer Ihm. In Ihn setze ich mein Vertrauen und zu Ihm werde ich heimkehren.«Q. 13,30.

\section{Aspekte des Vertrauens}

Im Qur'an wird dieses Vertrauen einerseits in einem allgemeinen moralischen und spirituellen Sinn thematisiert wie in dem Vers:

Allah! Es ist kein Gott außer Ihm; und auf Allah sollen die Gläubigen vertrauen. $(64,13)$

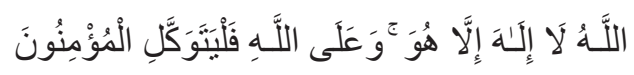

Aber auch in spezifischen Fällen äusserster Bedrängnis wie z.B. in Q. 3, 122:

Da verloren zwei Gruppen von euch beinahe den Mut, und Allah war beider Beschützer. Und auf Allah sollen sich die Gläubigen verlassen.

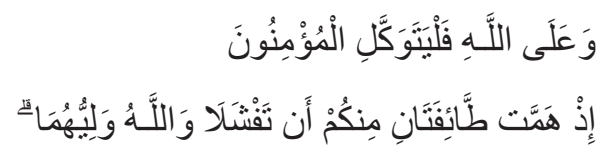

Dieser Vers verweist auf die Schlacht bei Badr, wo Muhammad mit einer kleinen Schar von Anhängern einem übermächtigen Feind gegenüberstand und doch siegte.

Und natürlich nicht zuletzt als Schutz nicht vor dem äusseren sondern vor dem inneren Feind, vor dem Bösen, vor Satan, z.B. in Q. 58, 10:

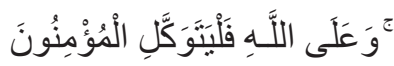

اللَّلَه

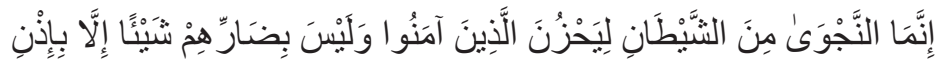

Die geheime Verschwörung rührt allein von Satan her, der die betrüben will, die gläubig sind; doch er kann ihnen nicht den geringsten Schaden zufügen, es sei denn mit Allahs Erlaubnis. Und auf Allah sollen die Gläubigen vertrauen. 
Sinn kann das Konzept eines unbedingten Vertrauens auf Gott aber nur haben, wenn damit zugleich das Konzept von der Omnipotenz Gottes bejaht wird und auch dasjenige von der göttlichen Vorsehung, indem das Schicksal eines jeden Dings in Gottes Hand liegt. Oder anders gesagt: Der qur'anische Imperativ, absolutes Vertrauen in Gott zu setzen und als logische Folge Gott als alleiniger Hüter und Beschützer in allen Lebenslagen anzunehmen, bedingt die Annahme der uneingeschränkten Schöpfungs- und Bestimmungsmacht Gottes. Machtvoll ausgedrückt im Qur'an in Stellen wie lā hawla wa lā quwwata illah billāh, (es gibt keine Macht und keine Stärke ausser bei Gott), welches von Gläubigen auch als eine Art Beschwörungsformel in grosser Bedrängnis gebraucht wird.

Oder in der Aussage, welche im Qur'an 35 mal vorkommt, nämlich: Gottes ist die Macht der Bestimmung über jedes Ding (Q. 2, 20):

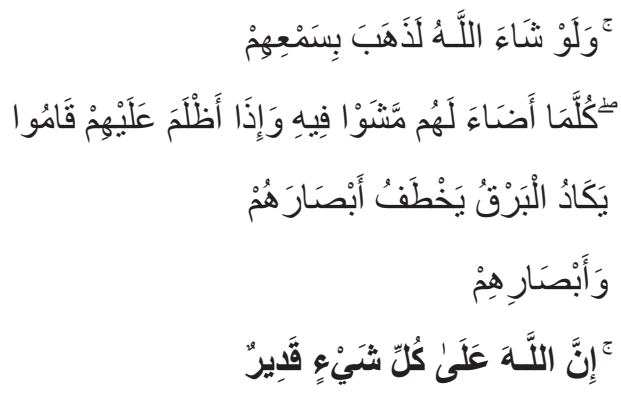

Der Blitz raubt ihnen beinahe ihr Augenlicht: Sooft er ihnen Licht gibt, gehen sie hindurch, und wenn es dunkel um sie wird, so bleiben sie stehen. Und wenn Allah wollte, hätte Er ihnen gewiß Gehör und Augenlicht genommen. Wahrlich, Allah ist über alle Dinge mächtig.

Aufs engste mit diesen Konzepten verknüpft ist auch das Vorstellung von Sabr, was - vor allem im qur'ānischen Kontext - mit Ausdauer oder Durchstehvermögen aber auch mit Selbstbeherrschung übersetzt werden könnte.

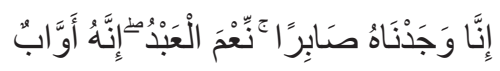


Wahrlich, Wir fanden ihn geduldig. (Er war) ein vortrefflicher Diener; stets wandte er sich (Allah) zu. 38.44.

Die Rede ist hier vom qur'anischen Ayyūb, dem biblischen Hiob.

Eine besondere Rolle spielte das Konzept des Tawakkul in Verbindung mit dem Konzept der Einzigkeit Gottes Tawhīd im Sufismus, der mystischen Ausrichtung des Islam. Darauf kann aber an dieser Stelle aus Zeitgründen ebenso wenig eingegangen werden wie auf die Auswirkungen dieser theologischen Konzepte auf Staat und Gesellschaft im Laufe der Geschichte.

\section{Misstrauen gegen den Islam}

Das Misstrauen gegen den Islam wiederum bildet eine Grundkonstante in der Beziehung zwischen dem christlichen Abendland und der islamischen Welt. Es ist das Misstrauen gegenüber einem Konkurrenten in Bezug auf Weltanschauung und Machtanspruch. Das christliche Abendland vermochte den Islam und die Muslime bis in die Neuzeit mit wenigen Ausnahmen nie wirklich anders zu sehen, als Herausforderung und Infragestellung des eigenen Wahrheits- und Geltungsanspruchs. Daran änderte auch die Tatsache nichts, dass die beiden Religionen und Kulturen von Anbeginn an in hohem Masse voneinander profitierten und sich gegenseitig befruchteten.

Das Misstrauen und die Dämonisierung des Islam haben eine lange Tradition und es war ein weiter Weg bis sich die katholische Kirche in der "Erklärung über das Verhältnis der Kirche zu den nichtchristlichen Religionen« Nostra aetate des 2. Vatikanischen Konzils von 1962-65, erstmals offiziell und vorsichtig positiv zum Islam und den Muslimen äusserte.

"Mit Hochachtung betrachtet die Kirche auch die Muslime, die den alleinigen Gott anbeten, den lebendigen und in sich seienden, barmherzigen und allmächtigen, den Schöpfer des Himmels und der Erde, der zu den Menschen gesprochen hat. Sie mühen sich, auch seinen verborgenen Ratschlüssen mit ganzer Seele zu unterwerfen, so wie Abraham sich Gott unterworfen hat, auf den der islamische Glaube sich gerne beruft."

Für den Ende des 7. und Anfang des 8. Jahrhunderts lebenden Johannes von Damaskus war der Islam nichts anderes als eine il- 
legitime christliche Sekte. Petrus Venerabilis wiederum unternahm Anfang des 12. Jahrhunderts seine Übersetzung des Qur'ans auf dem Hintergrund der geistig-militärischen Auseinandersetzung mit dem Islam und betrachtete sie als "Projekt zur Widerlegung des Islam». Und nochmals ungefähr einhundert Jahre später galt der Islam Thomas von Aquin in einem seiner Hauptwerke, Summa contra gentiles, als Heidentum. Dabei blieb es weitgehend.

Der Reformator Luther unterschied sich in Sachen Islam nicht wesentlich von seinen katholischen Kontrahenten, wie eines seiner Kirchenlieder zeigt, in welchem Papsttum und Islam gleichermassen Teufelswerk sind:

\author{
Erhalt uns Herr bey deinem Wort, \\ Und steu'r des Papsts und Türcken Mord, \\ die Jesum Christum, deinen Son \\ wollten stürtzen von deinem Thron.
}

Allerdings hatte Luther die Muslime nicht immer so negativ gesehen. Bevor unter dem Eindruck des Vordringens der Osmanen in Europa bis vor Wien die negative Wahrnehmung dominierte, konnte er bei den Muslimen auch positive Seiten erkennen. IhrVerhalten erschien ihm in mancher Hinsicht vorbildlich, insbesondere was ihre religiöse Praxis hinsichtlich des Gebets oder des Fastens anging. Keinen guten Faden liess er freilich am Propheten des Islam, Muhammad. In dieser Hinsicht waren sich auch Luther und Jean Calvin einig, dem Muhammad als eines der zwei Hörner des Antichristen galt. An der zwiespältigen Haltung Luthers zeigt sich exemplarisch, wie geopolitische Ereignisse sich unmittelbar auf die individuelle Wahrnehmung auswirken, und das nicht erst seit dem 11. September 2001.

Diese grundsätzlich negative Sicht auf den Propheten des Islam änderte sich auch unter den Aufklärern nicht: Bei Voltaire gilt $\mathrm{Mu}-$ hammad nicht als Prophet, sondern als gewissenloser Machtmensch und Lüstling. In Sachen Aufklärung über den Islam ist es bei den Aufklärern also nicht so gut bestellt, wie man vielleicht erwarten würde. Oder mit den Worten des palästinensisch stämmigen Literaturwissenschaftlers Edward Said: Die säkularisierende Tendenz der Aufklärung habe die alten religiösen Muster des Mittelalters nicht einfach abgeschafft, vielmehr wurden sie "rekonstruiert, wieder 
angewendet; im säkularen Rahmen neu verteilt. ${ }^{1}$ Auf diesem Hintergrund wird die Sensibilität der Muslime vielleicht etwas verständlicher, wenn es um die Diffamierung des Propheten geht wie im Karikaturenstreit oder in diesem vor kurzem in Umlauf gesetzten Video aus den USA.

Mit der Säkularisierung glaubte man in Europa das Ende der Religion als gesellschaftlich relevanter Faktor gekommen, Religion sozusagen als ein Auslaufmodell der Geschichte. Folgerichtig wurde deshalb bis in die 1990er Jahre hinein die Präsenz von Musliminnen und Muslimen in Europa weder von Politik und Gesellschaft noch von der Wissenschaft zur Kenntnis genommen. Sie galten als Türken, Araber, Jugoslawen - Ausländer eben. Die Migrationsforschung beschäftigte sich zwar mit "Gastarbeitern" aus der Türkei oder Jugoslawien, betrachtete sie jedoch allein unter soziologischen Gesichtspunkten. Die Andersartigkeit in religiöser Hinsicht dieser Menschen wurde weitestgehend ausgeblendet. Die religiösen Einstellungen und Aktivitäten der Musliminnen und Muslime interessierten kaum und galten, wenn überhaupt, als Randerscheinung und bar jeglicher Relevanz für die Mehrheitsgesellschaft. Höchstens konnte man in einer Lokalzeitung $\mathrm{ab}$ und zu eine Notiz lesen des Inhalts, Herr X. sei ein aufgeklärter - wahlweise auch liberal oder modern genannter - Muslim, da er ja einem Bier nicht abgeneigt sei. Oder die Freundschaft der Tochter mit einem Muslim wurde im Bekanntenkreis entschuldigt mit der Bemerkung, der Schwiegersohn in spe sei zwar Muslim aber, Gott sei Dank, kein praktizierender, wobei das Alkoholtrinken immer ein Schlüsselindiz war.

Diese Wahrnehmung bzw. Nicht-Wahrnehmung in religiöser Hinsicht änderte sich erstmals 1989 anlässlich der Rushdie-Affäre, als Muslime insbesondere in Grossbritannien in grosser Zahl aus religiösen Gründen gegen die Veröffentlichung der Satanischen Verse protestierten und ein Verbot des Buches verlangten. Auch den Muslimen wurde ihr religiöses Anderssein bzw. dessen rechtliche Konsequenzen nun erstmals bewusst: Die Rushdie-Affäre brachte ihnen nämlich mit aller Deutlichkeit zu Bewusstsein, dass britische Blasphemie-Gesetze ausschliesslich die christliche Religion schützen.

Als viele Musliminnen und Muslime in Grossbritannien 1991 gegen den ersten Golf-Krieg opponierten, wurde ihre Opposition nicht

1 Edward W. Said, Orientalismus. [Orientalism, 1978], Frankfurt a.M. 1981. 
als demokratisches Recht auf freie Meinungsäusserung interpretiert, sondern sie galten plötzlich als potenziell Subversive, als Fünfte Kolonne, als Illoyale einem Staat gegenüber, der sich im Kriegszustand befand. ${ }^{2}$ Obwohl sich die Aufregung nach und nach wieder etwas legte, blieb als gefährliche Hinterlassenschaft ein allgemeines Misstrauen gegenüber den Muslimen zurück.

Der grosse Bruch in der Wahrnehmung aber erfolgte im Gefolge des 11. September 2001. Aus Türken, Araber, ex-Jugoslawen, Pakistani wurden praktisch über Nacht "Muslime". Nun nahm man sie plötzlich auch in der Schweiz wahr als große (für viele zu große), fremde, bedrohliche Masse. Und mit jedem Anschlag, der irgendwo auf der Welt von muslimischen Gewalttätern verübt wurde, wuchs für viele Schweizerinnen und Schweizer das Gefühl einer Bedrohung durch diese unheimlichen Nachbarn, von denen man nichts wusste, aber eigentlich auch nichts wissen wollte. Und plötzlich war es wieder da, das seit dem Mittelalter im kollektiven Unterbewusstsein des christlichen Abendlandes schlummernde Bild vom Islam als gewalttätiger Religion und der "Türkengefahr«, also der Bedrohung Europas durch die Türken oder eben Muslime. Viele Muslime, die vorher problemlos mit ihren schweizerischen Nachbarn zusammengelebt hatten und von diesen Gewalttaten ebenso schockiert waren, sahen sich plötzlich mit einer Mauer des Misstrauens und der Ablehnung konfrontiert. Und noch besorgniserregender wurde die Gewohnheit, sie bei jeder Gelegenheit summarisch als "Fundamentalisten" wenn nicht gar »Terroristen« zu brandmarken.

Damit entstand der Zwang, sich dauernd erklären, rechtfertigen, abgrenzen zu müssen, zu beweisen, dass man ein "guter Muslim» war. Man war nun Muslim, wurde zum Muslim gemacht, der sich primär über seine Religion zu bestimmen hat.

Unterstützt und legitimiert wurde dieser Diskurs durch die Thesen von Samuel Huntington, die er in seinem Buch Kampf der Kulturen (The Clash of Civilizations) aufstellte. Eine seiner Hauptthesen in Bezug auf den Islam und die Muslime besagte, dass die religiöse Identität bei Muslimen dominant sei. Muslime könnten nicht zwischen privater Religion und öffentlichem Recht unterscheiden. Daher könnten nur Individuen, die Schlüsselelemente des islamischen Glaubens zurückwiesen, als vertrauenswürdige Gesprächspartner für demokratische Gesellschaften in Frage kommen. Eine These,

2 Philip Lewis, Islamic Britain, London 1994, 5. 
die noch heute im offiziellen Dialog mit Muslimen zumindest mitschwingt. Eine zweite Hauptthese betraf die Konkurrenz von aufgeklärtem Christentum (in Gestalt des Westens) und Islam um die globale Vormachtstellung. Hierbei präsentierte Huntington den Islam als monolithisch und unterstellte diesem so konstruierten Block ein Streben nach Vormachtstellung und Weltherrschaft. Muslime hätten nichts anderes im Sinn, als die Demokratie durch ein islamisches Rechtsystem, die "Scharia«, zu ersetzen.

Diese Thesen von Huntington blieben natürlich nicht unwidersprochen. Dennoch bildete sich ein Diskurs heraus, der bis heute anhält: »Indem politische, gesellschaftliche und soziale Phänomene zunehmend mit `der Religion ‘ der anderen verknüpft werden, können eigene Anteile an diesem Phänomen und am problematischen Verhältnis zueinander geleugnet werden", meint die Sozialwissenschaftlerin Iman Attia. Die Lage der Anderen wird mit deren 'Kultur begründet, die wesentlich durch sihre Religion` geprägt sei; >der Islamı sei für desolate Zustände verantwortlich und bilde eine Gefährdung. « ${ }^{3}$

Als Nebenwirkung dieses Diskurses entstand die innermuslimische Diskussion darüber, ob und wer und unter welchen Bedingungen denn eigentlich ein Muslim und was der "wahre" Islam sei. Diese Diskussion ist trotz relativ einfacher religionsrechtlicher Definition in vollem Gange. Darüber diskutiert wird insbesondere unter jüngeren Musliminnen und Muslimen in Internet-Foren, sozialen Medien und Chat-Rooms.

$\mathrm{Zu}$ diesen innermuslimischen Sichtweisen und Definitionen kommen nun noch die unterschiedlichsten Aussensichten hinzu:Aus Behördensicht sowohl in der Schweiz als auch in Deutschland galt bis vor kurzem als Muslim, wer aus einem muslimischen Land stammte oder einer bestimmten Ethnie angehörte. Mit Ausnahme der Volkszählung 2000, die eine Selbstdeklaration als Muslim ermöglichte, erfolgte die Ermittlung der Anzahl in der Schweiz lebender Muslime für die offizielle Statistik aufgrund der Herkunft und nicht des religiösen Bekenntnisses oder der Glaubenspraxis des Individuums und auch nicht aufgrund der Zugehörigkeit zu einem Moscheeverein. Nicht erfasst werden bei einem solchen Vorgehen Konvertiten, naturalisierte Schweizer Muslime und Kinder aus binationalen oder bireligiösen Ehen. Letztlich geben diese offiziellen Zahlen also

3 Iman Attia, Die "westliche Kultur« und ihr Anderes, Bielefeld 2009. 
lediglich Auskunft über die vermutete Anzahl der in der Schweiz oder Deutschland lebenden Menschen mit einem islamischen Hintergrund; die kulturelle Vielfalt, die unterschiedlichen Religionsauffassungen oder religiösen Praxen werden dabei ausgeblendet.

Das Muslimsein wird staatlicherseits auch dann definiert, wenn es um den Dialog mit "Vertreterinnen und Vertretern" der Muslime in der Schweiz oder Deutschland geht, sei es in der Deutschen Islamkonferenz oder dem Schweizer Muslimdialog. Der Islamwissenschaftler Frank Peter sieht in der Zusammenstellung der Deutschen Islamkonferenz ein solches Bestreben, die Grenzen der "muslimischen Gemeinschaft" in Deutschland zu bestimmen: "Die Zusammensetzung der Konferenz und die Auswahl der muslimischen Teilnehmer durch das Innenministerium stellt einen Versuch dar, einerseits das einzugrenzen, was als `Muslimische Gemeinschaft Deutschlands`zu gelten hat und andererseits welchen Qualifikationen diejenigen zu genügen haben um autorisiert zu sein, für diese Gemeinschaft zu sprechen." (»The convening of the conference and the selection of Muslim members by the ministry itself constituted a major attempt to define both the boundaries of Germany's >Muslim community and the qualifications of those authorized to speak for it.« ${ }^{4}$ Dies hat zur Folge, dass sich religiös positionieren muss, wer an diesem Dialog teilnehmen und im Diskurs wahrgenommen werden will. Sehr deutlich war dieses Phänomen auch in der Schweiz nach der Annahme der Anti-Minarett-Initiative zu beobachten. Es entfaltete sich ein behördlicher Aktivismus, der zu einer Vielzahl von Dialogplattformen führte. Personen, die sich zuvor nie an Diskussionen zum Islam oder den Muslimen in der Schweiz beteiligt hatten, wurden nun behördlicherseits plötzlich zu Vertreterinnen undVertretern der Muslime auserkoren.

Im akademischen Diskurs gibt es weitere Definitionen. Die islamwissenschaftliche Literatur nimmt im Allgemeinen die Zugehörigkeit zum Islam als gegeben hin. Gudrun Krämer moniert, dass für traditionelle Orientalisten »Muslime [...] allein dadurch hinreichend definiert [sind], dass sie Muslime sind, wobei es wenig Unterschied

\footnotetext{
4 Frank Peter, Welcoming Muslims into the nation. Tolerance, politics and integration in Germany, in: Jocelyne Cesari (Hg.), Muslims in the West after 9/11, London/ New York 2010, 120.
} 
macht, ob sie in Kuala Lumpur, Kairo oder Karachi leben. Sie sind durch ihre Zugehörigkeit zum Islam determiniert. $\|^{5}$

Die öffentlichen Debatten über Muslime folgen zunehmend einem Muster, welches der französische Islamwissenschaftler Olivier Roy als "Neoethnisierung" bezeichnet. Er meint damit die Konstruktion einer muslimischen Gemeinschaft (community) von innen und aussen, vergleichbar mit der Konstruktion von ethnischen Gemeinschaften als "construction of an ethnic group which previously did not exist as such. $\ll^{6}$ Dieser Prozess geht einher mit der Abwertung der regionalen bzw. ethnischen Wurzeln und der Aufwertung einer Verbindung zur Religion Islam in der diskursiven Praxis. Die Herkunftskultur ist nicht länger relevant, sondern wird ersetzt durch den Bezug auf eine neu konstituierte Gruppe: nämlich die Gemeinschaft der Muslime. Die Zugehörigkeit zu dieser Gemeinschaft werde als unabhängig vom Glauben und den religiösen Praktiken des Menschen gesehen, vielmehr richte sie sich nach einem sset of cultural patterns that are assumed to be inherited. «"

\section{Zum Muslim gemacht werden}

Es versteht sich von selbst, dass die Wahrnehmung ihrer Religion und Kultur durch die Mehrheitsgesellschaft insbesondere für die Identitätsbildung von jungen Musliminnen und Muslimen Konsequenzen hat. Die Mediendiskurse zum Thema "Wir und die Muslime«, das weit verbreitete und gesellschaftlich akzeptierte Klischee vom Islam als gewalttätiger, blindwütiger und vernunftwidriger Religion der Unfreiheit sowie das Gefühl, permanent unter Verdacht zu stehen, sind nicht gerade hilfreich für den Aufbau einer positiven muslimischen Identität. Sie führen letztlich zu einer apologetischen und polemischen Haltung. Apologetisch, weil sich die Muslime gegen die Unterstellungen und Verdächtigungen verteidigen müssen, polemisch, weil sie dabei dazu neigen, Vorwürfen auszuweichen oder sie zurückzugeben, statt die Diskussion auf einer inhaltlich-sachlichen Ebene zu führen.

5 Gudrun Krämer, zit. nach: Riem Spielhaus, Wer ist hier Muslim? Die Entwicklung eines islamischen Bewusstseins in Deutschland zwischen Selbstidentifikation und Fremdzuschreibung, Würzburg 2011, 95.

${ }^{6}$ Olivier Roy, Globalized Islam. The Search for a New Ummah. Columbia University Press, New York 2004, 124.

7 Ebd. 
Der gesamte Diskurs um Musliminnen und Muslime in Europa zeigt, dass die Bedeutung religiöser Zugehörigkeit derzeit deutlich steigt. Die Etablierung von Identitäten, die eine religiöse Zuordnung ausser Acht lässt, scheint dabei für Migranten und Migrantinnen und deren Nachkommen aus mehrheitlich muslimischen Ländern kaum mehr möglich. Nach Einschätzung der deutschen Islamwissenschaftlerin Riem Spielhaus führt »die von muslimischer und nicht-muslimischer Seite betriebene Konstruktion der Gruppe der Muslime zu Selbst- und Fremdwahrnehmung in den gegensätzlichen Kategorien >Ihrı und 'Wir', sunsere Gemeinschaft und seure Gemeinschaft auf beiden Seiten. Andere Identitäten, wie soziale, berufliche, lokale oder nationale scheinen weniger wichtig und durch die (teils lediglich angenommene) religiöse Zugehörigkeit in den Hintergrund gedrängt.$^{8}$ Die deutsch-türkische Publizistin Hilal Sezgin hat einmal in einem Interview für das Recht plädiert, über die eigene Religion zu schweigen. Also nicht ständig als Mitglied einer bestimmten Religion adressiert zu werden oder sich erklären zu müssen. Das Recht, selbst zu bestimmen, in welchen Kontexten die eigene Religionszugehörigkeit von Bedeutung ist und wann nicht.

Diese angeordneten Dialoge wie die Deutsche Islamkonferenz oder der Schweizer Islamdialog können als vertrauensbildende Massnahmen verstanden werden und sind vermutlich auch als solche gedacht. Vertrauen lässt sich aber nicht anordnen und ob sich das geschwundene Vertrauen zwischen Mehrheit und muslimischer Minderheit damit wieder herbeireden lässt, darf bezweifelt werden.

— Dr. h.c. lic. phil. Rifa'at Lenzin arbeitet als freischaffende Islamwissenschaftlerin und Publizistin. Sie ist am Zürcher Lehrhaus zuständig für den Bereich Islam.

8 Riem Spielhaus, Wer ist hier Muslim? Die Entwicklung eines islamischen Bewusstseins in Deutschland zwischen Selbstidentifikation und Fremdzuschreibung, Würzburg 2011, 55 